\title{
Editorial: Advances in Understanding NeuroHIV Associated Changes in Neuroimmune Communication in the Combined Anti-retroviral Therapy (cART) Era
}

\author{
Peter J. Gaskill ${ }^{*}$, Jerel Adam Fields ${ }^{2}$, Dianne T. Langford ${ }^{3}$, Kelly L. Stauch ${ }^{4}$ and \\ Dionna W. Williams ${ }^{5}$
}

${ }^{1}$ Department of Pharmacology and Physiology, Drexel University College of Medicine, Philadelphia, PA, United States, ${ }^{2}$ Department of Psychiatry, University of California San Diego, La Jolla, CA, United States, ${ }^{3}$ Department of Neuroscience, Lewis Katz School of Medicine, Philadelphia, PA, United States, ${ }^{4}$ Department of Neurological Sciences, University of Nebraska Medical Center, Omaha, NE, United States, ${ }^{5}$ Department of Molecular and Comparative Pathobiology, School of Medicine, Johns Hopkins University, Baltimore, MD, United States

Keywords: neuroAIDS, neuroimmunity, neuropathogenesis, neurotransmission, CART, neuroHIV

\section{Editorial on the Research Topic}

\section{OPEN ACCESS}

Edited and reviewed by: Hans-Peter Hartung, Heinrich Heine University of

Düsseldorf, Germany

*Correspondence:

Peter J. Gaskill pjg63@drexel.edu

Specialty section:

This article was submitted to Multiple Sclerosis and

Neuroimmunology,

a section of the journal

Frontiers in Neurology

Received: 23 August 2021 Accepted: 08 September 2021

Published: 05 October 2021

Citation: Gaskill PJ, Fields JA, Langford DT, Stauch KL and Williams DW (2021)

Editorial: Advances in Understanding NeuroHIV Associated Changes in Neuroimmune Communication in the Combined Anti-retroviral Therapy (CART) Era. Front. Neurol. 12:763448. doi: 10.3389/fneur.2021.763448
Advances in Understanding NeuroHIV Associated Changes in Neuroimmune Communication in the Combined Anti-retroviral Therapy (cART) Era

It has been almost 40 years since the first cases of AIDS were reported in Morbidity and Mortality Weekly in the fall of 1981 (1). During that time, almost 80 million people have been infected and more than 36 million people have died from HIV, with 1.5 million new infections and 680,000 deaths occurring in 2020. Despite these alarming numbers, there has been significant progress in controlling this pandemic as HIV infections and deaths have decreased 50-60\% from their peaks in 1997 and 2004, respectively. This is largely due to the development and implementation of antiretroviral therapy (ART). In 2020, around two thirds of people living with HIV (PLWH) were treated with ART to suppress viral replication (2-6). Widespread access to ART has brought us to an inflection point in the pandemic, transforming HIV from a terminal diagnosis to a chronic condition.

Unfortunately, chronic infection and ART treatment have led to a variety of new health challenges, including long-term neurological dysfunction. In the absence of ART, PLWH showed rampant neuroinflammation and significant neuronal death associated with high rates of dementia. With ART, the neuropathology associated with HIV infection has become both more subtle and more complex. Importantly, even with suppression of viral replication to below the level of detection, neuropathological changes, minor neurocognitive dysfunction, depression, and other neuropsychiatric adverse events (NPAE) remain prevalent (5, 7-15), suggesting that the etiology of these conditions is not solely derived from viral replication. As Spagnolo-Allende and Gutierrez discuss in their review of cerebrovascular complications in neuroHIV, HIV proteins and associated neuroinflammation can initiate and/or exacerbate multiple types of vascular complications. Distinct phenotypes of vascular insults are associated with the presence or absence of ART in PLWH, so further study in this area is important given the growing incidence of cerebrovascular disease in PLWH on ART, particularly older individuals. 
Neuronal health and function are maintained by complex, bidirectional interactions among neurons, astrocytes, microglia, and central nervous system (CNS) macrophages (16-18). Data suggest that neuroHIV stems, at least partially, from disruption of this communication, impairing neurotransmission, synapse formation/dissolution and neuroimmune communication. Some studies suggest that antiretroviral (ARV) drugs themselves may contribute to these changes in neuroimmune communication and neurological dysfunction (19-22). The data from Tripathi et al. support this possibility, showing that in both isolated rodent microglia and in HIV-1 transgenic rats, exposure to ART mediates microglial activation through oxidative stress-mediated lysosomal dysfunction. George et al., also demonstrate that antiretroviral drugs dolutegravir, emtricitabine, and efavirenz induce cell-type specific changes in ATP generation and mitochondrial respiration in epithelial and microglial cell lines, suggesting metabolic changes may contribute ARV toxicity in specific cell types. Dastgheyb et al. compared neurocognitive function between 929 virally suppressed women living with HIV and 717 HIV-uninfected women, identifying distinct neuropsychological profiles associated with both demographic and clinical variables, including the use of specific ARVs. Together, these data indicate more in-depth analyses of specific impacts of distinct ARVs both in vitro in discrete cell types and in humans in conjunction with analyses of a larger combination of variables could help to identify and/or predict neuropathological and neurocognitive response patterns to specific ART regimens.

Currently, the spread of HIV is increasingly driven by marginalized populations, including transgender women, sex workers, men who have sex with men and individuals using injection drugs. In each of these groups, the risk of HIV infection is 25 to 35 times higher than that in the general population (3). These populations have a high prevalence of substance use disorders (23-28) and hepatitis C (HCV) (2932 ), so it is critical to evaluate their impact on neuroimmune communication, neuropathogenesis and neurological function. Paul et al. analyzed the neurocognitive response to ART in PLWH with co-occurring HCV infection, showing that both mono-HIV infected, and co-HIV/HCV infected individuals had significant neurocognitive improvement in response to ART. Notably, a subgroup of co-infected individuals with higher HIV plasma viral load and lower plasma $\mathrm{CD} 4^{+} \mathrm{T}$ cell count at baseline showed persistent motor deficits. In contrast, Matt et al. show that exposure to dopamine concentrations induced by the use of addictive drugs $(33,34)$ alters the efficacy of the CCR5 antagonist Maraviroc by increasing CCR5 expression on the macrophage surface. Cisneros et al., show that methamphetamine mediated activation of trace amino acid receptor 1 (TAAR1) triggers multiple signaling pathways in human astrocytes, regulating the expression of the glutamate receptor EAAT-2 through activation of CAMKII and $\mathrm{Ca}^{2+}$ release, and phosphorylating CREB via both the $\mathrm{Ca}^{2+}$ release and cAMP pathways. These data support substantial research indicating that both dopamine and substances of abuse can potentially exacerbate HIV infection and dysregulate associated cellular processes in the CNS.
While the above studies suggest that substances of abuse could exacerbate neuroHIV, other studies on abused substances suggest novel therapeutic activity for different drugs or neurotransmitter systems. In primary rodent neurons, League et al., show that blocking the activity of monoacylglyerol lipase (MAGL) reduces the neurotoxic effects of Tat. As MAGL drives metabolism of the cannabinoid receptor $\left(\mathrm{CB}_{1} \mathrm{R}\right)$ agonist, 2 -arachidonoylglycerol, these data support the idea of using the endocannabinoid system as a target for neurotherapeutic adjuvants in ART-treated PLWH. Lin et al. show that the benzodiazepine, alprazolam (Xanax), alters the activity of the transcription factor RUNX1 and STAT5. Recent data show that benzodiazepines are overprescribed to PLWH and are associated with neurocognitive deficits, so these data suggest alprazolam may influence neuroinflammation and provide a mechanism underlying prior studies showing alprazolam can reactivate latent HIV. Han et al. use the iTat rodent model to show that Tat expression increases functional and structural indices of motor and sensory neuropathy, dysregulating the expression of proteins in the electron transport chain and the mitochondria. These effects were blocked by treatment with the muscarinic receptor 1 antagonist pirenzepine, which promotes mitochondrial biogenesis, suggesting the involvement of the muscarinic system in HIV distal sensory polyneuropathy. In another study using the iTat model, Joshi et al., compare acute vs. prolonged Tat induction and show prolonged Tat induction reduced locomotor activity and caused a small but significant increase in the ratio of MMP to TIMP1, while the acute induction of Tat reduced IL-6 mRNA expression.

The studies from this special collection reinforce that the development of neuroHIV is multi-factorial, driven by altered interactions among distinct types of CNS cells and interconnecting neurotransmitter, signaling and metabolic pathways. These data also collectively show that neuroHIV can be exacerbated by a variety of comorbidities and other factors including ART. Future studies should continue to evaluate the discrete impacts of ART and comorbidities, like substance abuse and HCV, as well as the combined effects of these conditions and their capacity to drive the growing number of complications associated with neuroHIV.

\section{AUTHOR CONTRIBUTIONS}

PJG, JAF, DTL, KLS, and DWW wrote the editorial and invited authors to participate in the collection. All authors contributed to the article and approved the submitted version.

\section{FUNDING}

This work was supported by grants from the National Institutes of Drug Abuse (DA039005 and DA049227 to PJG, DA037830 to DTL, DA044838 and DA052859 to DWW), National Institutes of Mental Health (MH115819 to JAF, MH107340 to DTL, MH062261 pilot funding to KLS), National Institutes of Aging (AG066215 to JAF) and the W.W. Smith Charitable Trust (A2003 to PJG). 


\section{REFERENCES}

1. CDC. Pneumocystis pneumonia - Los Angeles. MMWR. (1981) 30:250-2.

2. UNAIDS. Fact Sheet 2021 (2021).

3. UNAIDS. CONFRONTING INEQUALITIES Lessons for pandemic responses from 40 years of AIDS. Joint United Nations Programme on HIV/AIDS, Geneva (2021)

4. Cihlar T, Fordyce M. Current status and prospects of HIV treatment. Curr Opin Virol. (2016) 18:50-6. doi: 10.1016/j.coviro.2016.03.004

5. Saylor D, Dickens AM, Sacktor N, Haughey N, Slusher B, Pletnikov M, et al. HIV-associated neurocognitive disorder-pathogenesis and prospects for treatment. Nat Rev Neurol. (2016) 12:234-48. doi: 10.1038/nrneurol.2016.27

6. Boender TS, Smit C, van Sighem A, Bezemer D, Ester CJ, Zaheri S, et al. AIDS therapy evaluation in the Netherlands (ATHENA) national observational HIV cohort: cohort profile. BMJ Open. (2018) 8:e022516. doi: 10.1136/bmjopen-2018-022516

7. Eggers C, Arendt G, Hahn K, Husstedt IW, Maschke M, NeuenJacob E, et al. HIV-1-associated neurocognitive disorder: epidemiology, pathogenesis, diagnosis, and treatment. J Neurol. (2017) 264:1715-27. doi: 10.1007/s00415-017-8503-2

8. Bell JE. An update on the neuropathology of HIV in the HAART era. Histopathology. (2004) 45:549-59. doi: 10.1111/j.1365-2559.2004.02004.x

9. Everall IP, Hansen LA, Masliah E. The shifting patterns of HIV encephalitis neuropathology. Neurotoxicity Res. (2005) 8:51-61. doi: 10.1007/BF03033819

10. Saylor D, Dickens AM, Sacktor N, Haughey N, Slusher B, Pletnikov M, et al. HIV-associated neurocognitive disorder - pathogenesis and prospects for treatment. Nat Rev Neurol. (2016) 12:309. doi: 10.1038/nrneurol.2016.53

11. Becker JT, Sanders J, Madsen SK, Ragin A, Kingsley L, Maruca V, et al. Subcortical brain atrophy persists even in HAART-regulated HIV disease. Brain Imaging Behav. (2011) 5:77-85. doi: 10.1007/s11682-011-9113-8

12. Ipser JC, Brown GG, Bischoff-Grethe A, Connolly CG, Ellis RJ, Heaton RK, et al. HIV infection is associated with attenuated frontostriatal intrinsic connectivity: a preliminary study. J Int Neuropsychol Soc. (2015) 21:203-13. doi: $10.1017 /$ S1355617715000156

13. Ortega M, Brier MR, Ances BM. Effects of HIV and combination antiretroviral therapy on cortico-striatal functional connectivity. AIDS. (2015) 29:703-12. doi: 10.1097/QAD.0000000000000611

14. Alakkas A, Ellis RJ, Watson CW, Umlauf A, Heaton RK, Letendre S, et al. White matter damage, neuroinflammation, neuronal integrity in HAND. $J$ Neurovirol. (2019) 25:32-41. doi: 10.1007/s13365-018-0682-9

15. Valcour V, Sithinamsuwan P, Letendre S, Ances B. Pathogenesis of HIV in the central nervous system. Curr HIV/AIDS Rep. (2011) 8:54-61. doi: 10.1007/s11904-010-0070-4

16. Dantzer R, Kelley KW. Psychoneuroimmune phenomena: neuroimmune interactions. In: Pfaff DW, Volkow ND, editors. Neuroscience $n$ the 21st Century. New York, NY: Springer (2016). p. 643-71.

17. Herz J, Filiano AJ, Smith A, Yogev N, Kipnis J. Myeloid cells in the central nervous system. Immunity. (2017) 46:943-56. doi: 10.1016/j.immuni.2017.06.007

18. Liddelow SA, Marsh SE, Stevens B. Microglia and astrocytes in disease: dynamic duo or partners in crime? Trends Immunol. (2020) 41:820-35. doi: 10.1016/j.it.2020.07.006

19. Lagathu C, Eustace B, Prot M, Frantz D, Gu Y, Bastard JP, et al. Some HIV antiretrovirals increase oxidative stress and alter chemokine, cytokine or adiponectin production in human adipocytes and macrophages. Antivir Ther. (2007) 12:489-500. doi: 10.1177/125965350701200407

20. Chen L, Al-Harthi L, Hu XT. Triumeq increases excitability of pyramidal neurons in the medial prefrontal cortex by facilitating voltagegated $\mathrm{Ca}(2+)$ channel function. Front Pharmacol. (2020) 11:617149. doi: $10.3389 /$ fphar.2020.617149

21. Shah A, Gangwani MR, Chaudhari NS, Glazyrin A, Bhat HK, Kumar A. Neurotoxicity in the post-HAART era: caution for the antiretroviral therapeutics. Neurotox Res. (2016) 30:677-97. doi: 10.1007/s12640-016-9646-0

22. Yuan NY, Kaul M. Beneficial and adverse effects of cART affect neurocognitive function in HIV-1 infection: balancing viral suppression against neuronal stress and injury. J Neuroimmune Pharmacol. (2021) 16:90112. doi: 10.1007/s11481-019-09868-9

23. Bourne A, Weatherburn P. Substance use among men who have sex with men: patterns, motivations, impacts and intervention development need. Sex Transm Infect. (2017) 93:342-6. doi: 10.1136/sextrans-2016-052674

24. Rosińska M, Gios L, Nöstlinger C, Berghe WV, Marcus U, Schink S, et al. Prevalence of drug use during sex amongst MSM in Europe: results from a multi-site bio-behavioural survey. Int J Drug Policy. (2018) 55:231-41. doi: 10.1016/j.drugpo.2018.01.002

25. Connolly D, Gilchrist G. Prevalence and correlates of substance use among transgender adults: a systematic review. Addict Behav. (2020) 111:106544. doi: 10.1016/j.addbeh.2020.106544

26. Strathdee SA, West BS, Reed E, Moazan B, Azim T, Dolan K, et al. Substance use and HIV among female sex workers and female prisoners: risk environments and implications for prevention, treatment, and policies. J Acquir Immune Defic Syndr. (2015) 69(Suppl. 2):S110-17. doi: 10.1097/QAI.0000000000000624

27. Reback CJ, Fletcher JB. HIV prevalence, substance use, and sexual risk behaviors among transgender women recruited through outreach. AIDS Behav. (2014) 18:1359-67. doi: 10.1007/s10461-013-0657-z

28. Roxburgh A, Degenhardt L, Larance B, Copeland J. Mental Health, Drug Use and Risk Among Female Street-Based Sex Workers in Greater Syndney. Sydney, NSW: National Drug and Alcohol Research Centre (2005).

29. Midgard H, Weir A, Palmateer N, Re III VL, Pineda JA, Macías J, et al. HCV epidemiology in high-risk groups and the risk of reinfection. J Hepatol. (2016) 65:S33-45. doi: 10.1016/j.jhep.2016.07.012

30. Goldenberg SM, Montaner J, Braschel M, Socias E, Guillemi S, Shannon K, et al. Dual sexual and drug-related predictors of hepatitis $\mathrm{C}$ incidence among sex workers in a Canadian setting: gaps and opportunities for scale-up of hepatitis C virus prevention, treatment, and care. Int J Infect Dis. (2017) 55:31-7. doi: 10.1016/j.ijid.2016.12.019

31. Hernandez CJ, Trujillo D, Sicro S, Meza J, Bella M, Daza E, et al. High hepatitis $\mathrm{C}$ virus seropositivity, viremia, and associated risk factors among trans women living in San Francisco, California. PLoS ONE. (2021) 16:e0249219. doi: 10.1371/journal.pone.0249219

32. Karimi SE, Bayani A, Higgs P, Bayat AH, Hemmat M, Ahounbar E, et al. Prevalence and high risk behaviours associated with HCV testing among people who inject drugs: a systematic review and Meta-analysis. Subst Abuse Treat Prev Policy. (2020) 15:64. doi: 10.1186/s13011-020-00306-1

33. Matt SM, Gaskill PJ. Where is dopamine and how do immune cells see it?: Dopamine-mediated immune cell function in health and disease. I Neuroimmune Pharmacol. (2020) 15:114-64. doi: 10.1007/s11481-019-09851-4

34. Di Chiara G, Imperato A. Drugs abused by humans preferentially increase synaptic dopamine concentrations in the mesolimbic system of freely moving rats. Proc Natl Acad Sci USA. (1988) 85:5274-8. doi: 10.1073/pnas.85. 14.5274

Conflict of Interest: The authors declare that the research was conducted in the absence of any commercial or financial relationships that could be construed as a potential conflict of interest.

Publisher's Note: All claims expressed in this article are solely those of the authors and do not necessarily represent those of their affiliated organizations, or those of the publisher, the editors and the reviewers. Any product that may be evaluated in this article, or claim that may be made by its manufacturer, is not guaranteed or endorsed by the publisher.

Copyright (c) 2021 Gaskill, Fields, Langford, Stauch and Williams. This is an openaccess article distributed under the terms of the Creative Commons Attribution License (CC BY). The use, distribution or reproduction in other forums is permitted, provided the original author(s) and the copyright owner(s) are credited and that the original publication in this journal is cited, in accordance with accepted academic practice. No use, distribution or reproduction is permitted which does not comply with these terms. 